\title{
Diseño, validez facial y de contenido del instrumento carga de la enfermedad crónica para el paciente - GPCP-UN
}

\author{
Beatriz Sánchez-Herrera ${ }^{1}$, cbsanchezh@unal.edu.co, Carrillo Gloria Mabel², Cárdenas Diana Carolina ${ }^{3}$, Alarcón Angie Alejandra ${ }^{3}$.
}

Enfermera. Master in Science of Science of Nursing. Profesora titular (p) Universidad Nacional de Colombia, Profesora Universidad de la Sabana. Enfermera. Profesora asociada. Universidad Nacional de Colombia. Sede Bogotá. Facultad de Enfermería.

Enfermera. Universidad Nacional de Colombia

Fecha de recepción febrero 23 de 2016 Fecha de correcciones junio 1 de 2016

Fecha de aceptación septiembre 30 de 2016 Fecha de publicación enero 31 de 2017

\section{Resumen}

Objetivo: Diseñar y realizar validación facial y de contenido del instrumento Carga de la Enfermedad Crónica para el Paciente. Método: Estudio descriptivo desarrollado entre los años 2012 - 2015, en las siguientes fases: 1) Revisión de la literatura. 2) Recuento y evaluación y de síntomas, quejas y preocupaciones expresadas por 1850 pacientes con seguimiento de un año. 3) Construcción del instrumento 4) Prueba de comprensibilidad y ajuste semántico 5) Pruebas de validez facial y de contenido con expertos. Resultados. El instrumento Carga de la Enfermedad Crónica para el Paciente - GCPC-UN consta de 49 ítems. Cada situación que se asocia con la enfermedad es medida en términos de la frecuencia, la intensidad y el nivel en que es considerada amenazante o peligrosa. Conclusiones. El Instrumento permite valorar integralmente el impacto que la situación de enfermedad crónica genera a la persona con la enfermedad. Los resultados de sus pruebas de comprensibilidad, validez facial y contenido, avalan su aplicación en el contexto colombiano y son base para nuevas investigaciones en el campo. Palabras clave. Enfermedad crónica, signos y síntomas, costo de la enfermedad, rol del enfermo.

Copyright ( $\odot$ Facultad de Ciencias de la Salud de la Universidad Tecnológica de Pereira. 1995-2017. Todos los derechos reservados *.
Design, facial and content validation of the instrument "Burden of chronic illness for the patient - gcpc-UN"

\section{Abstract}

Objective: To design and perform facial and content validation of the instrument Burden of Chronic Illness for the Patient - GCPC UN. Method: Descriptive study developed between the years 2012 - 2015, in the following phases: 1) Review of the literature. 2) Count and evaluation of symptoms, complaints and concerns expressed by 1850 patients with a follow up of one year. 3) Construction of the instrument 4) Proof of comprehensibility and semantic adjustment 5) facial and content validation with experts. Results: The instrument "Burden of Chronic Illness for the Patient - GCPC- UN" consists of 49 items divided into three dimensions: the psychological and spiritual suffering, with 15 items; the discomfort and physical distress, with 19 items; and the altered cultural and family patterns with 15 items; 1 final question that gives options for other problems was included. Each situation associated with the disease is measured in terms of frequency, intensity and the level in which it is considered threatening or dangerous. Conclusions: The Instrument "Burden of Chronic Illness for the Patient - GCPC UN" can fully assess the impact of chronic Illness for the person with a chronic disease. The results of the tests of comprehensibility, as well as the facial and content validity tests support the instrument "Burden of Chronic Illness for the Patient - GCPC UN" for its application in the Colombian context and are the basis for further research in the field.

Keywords: Chronic disease, signs and symptoms, cost of illness, the sick role. Copyright () Facultad de Ciencias de la Salud de la Universidad Tecnológica de Pereira. 1995-2017. All rights reserved .

\section{Introducción}

Con el inesperado incremento de la edad y de la enfermedad crónica (EC) en el mundo[1], los sistemas de salud se han visto comprometidos a luchar contra una potencial amenaza a la estabilidad socioeconómica y al funcionamiento efectivo de los servicios de salud [2] por la demanda aumentada en la atención, el limitado tiempo de consulta, el esfuerzo generado en pacientes y cuidadores para el manejo adecuado de los tratamientos, la medicación, la dieta, el ejercicio, el monitoreo y las repercusiones de la experiencia de la cronicidad en el ámbito personal [3].

En consecuencia, los sistemas de salud han comenzado a sentir la necesidad de sumar a la atención de calidad, metas relativas a la reducción de los costos por paciente [4], en muchos casos el sistema no se encuentra preparado para asumir dichos costos y mucho menos para facilitar la vivencia de quienes se ven afectados por estas situaciones.

Al tratar de comprender la experiencia de las personas con EC, se ha señalado que dicha enfermedad se relaciona de manera importante con la calidad de vida [5,6] con la autopercepción de salud vista como la ayuda recibida y la satisfacción de las personas con su existencia [7]. En tal sentido, se ha planteado que la enfermedad además del padecimiento físico tiene un significado social [8] y un costo económico [9] y por ello se ha valorado la autopercepción de la carga del cuidado en quienes viven con una EC [10], en quienes enfrentan una situación de muerte inminente [11], y en quienes sienten que interfieren el proyecto de vida de su cuidador [12].

La mayoría de investigaciones actuales direccionan la medición de la carga de enfermedad a aspectos económicos y humanísticos [13-14]. No obstante, la percepción de la EC por parte del paciente ha sido también valorada mediante el desarrollo técnico científico para la medición de síntomas desagradables señalando la importancia de abordarlos de forma completa [15].

Es evidente en este panorama la falta de una herramienta que mire de forma integral cuál es la percepción de la carga de la EC para el paciente. Este trabajo buscó diseñar y realizar validación facial y de contenido del instrumento que valora la carga de la EC para el paciente a partir de su experiencia de vivir en esta situación.

\section{Materiales y métodos}

Estudio descriptivo que hace parte del trabajo multicéntrico de la Red Latinoamericana de Cuidado al Paciente Crónico (RED) y que fue desarrollado entre los años 2012 - 2015, en las siguientes cinco fases: 
1) Revisión de la literatura.

La revisión de la literatura se desarrolló bajo la metodología de Ganong (16) modificada por Sparbel, Anderson (17) y que es conocida como revisión integradora. Con base en ella, se clasificó y analizó la información derivada de investigación primaria sobre la carga de la EC para el paciente de manera sistemática a través de plantear la pregunta a responder y que hizo referencia a qué dice la literatura mundial de los últimos 20 años de 1994 - 2014, sobre la carga de la enfermedad crónica para el paciente. A partir de esta definición se establecieron los criterios de búsqueda. Con el empleo del metabuscador "Descubridor" del Sistema Nacional de Bibliotecas de la Universidad Nacional de Colombia que incluye las bases de datos Scielo; Medline With Full Text; Sciencedirect; Infotrac Health Reference Center Academic; Academic Onefile; Business Source Complete; British Library Ethos; Expanded Academic Asap; Health \& Wellness Resource Center; Psycarticles; General Onefile; Informit Health Collection; Econlit With Full Text; Library, Information Science \& Technology Abstracts; y Science In Context, se procedió a la búsqueda de los estudios bajo los criterios especificados de tiempo, con 20 años; idiomas inglés y español con descriptores DeCS y operadores boleanos AND y OR, atendiendo las siguientes fórmulas de búsqueda: carga OR costo AND síntomas OR quejas OR quejas y síntomas OR signos y síntomas AND Paciente OR Enfermo OR Persona con enfermedad AND enfermedad crónica OR padecimiento crónico AND escalas OR instrumentos OR test OR valoración; con sus respectivas traducciones al idioma inglés. Se procedió a la preparación de una matriz de análisis para recopilar los datos que incluyó la referencia, objetivo, método, resultados y aportes especiales para la comprensión del fenómeno estudiado. Una vez revisados los estudios se identificaron las pautas para el análisis e interpretación de datos y se hicieron la revisión y ajustes requeridos a la herramienta de recolección de los mismos. Con este ajuste, se procedió a la recopilación de la información, su análisis sistemático y la posterior discusión e interpretación de los datos y el reporte de los hallazgos.

2) Evaluación y recuento de síntomas, quejas y preocupaciones expresadas por los pacientes. Durante un año y con el apoyo de personal asistencial, estudiantes y cuidadores familiares vinculados a la RED, se llevó un registro sistemático de las quejas de los pacientes con EC para saber cuáles eran sus síntomas, preocupaciones, molestias, angustias, o dolencias. Para la identificación de las mismas, se elaboró un formato en físico y se registraron dichas quejas y demás expresiones de inquietud siempre que estas estuvieran relacionadas con la enfermedad, su tratamiento, manejo o consecuencias. Una vez escuchadas estas fueron registradas y tabuladas en una matriz diseñada para ese propósito. Los pacientes considerados se encontraban en diferentes escenarios que incluyeron hospitalización en servicios de alta y baja complejidad, consulta externa, comunidad y hogar. Se incluyeron pacientes de diferentes edades y género y con diferentes tipos de enfermedad incluyendo aquellas que exigen modificar el estilo de vida para mejorar el pronóstico, las que generan limitación, dolor y mutilación, las que alteran la socialización y las que generan una gran pérdida o enfrentamiento con la muerte.

3) Construcción del instrumento. A partir de un análisis sobre referentes teóricos para orientar el ejercicio, se seleccionaron los de la teoría de los síntomas desagradables de Lenz y Pugh [18] y la teoría del manejo de los síntomas que proponen Humpreys y cols. [19], que son complementarias entre sí. A partir de ello y teniendo en cuenta las escalas de uso clínico e investigativo para determinar preocupaciones, quejas y síntomas en personas con EC reportadas en la literatura, se llevó a cabo un análisis de la información suministrada por los pacientes generando los ítems y posterior agrupación en categorías. Se buscó al hacerlo mantener el significado que le dio el paciente a su queja.
4) Prueba de comprensibilidad y ajuste semántico. Este proceso consistió en la determinación de grado de comprensibilidad del instrumento, es decir el entendimiento y claridad de los ítems por parte de personas del común con diferentes niveles de escolaridad, estrato socioeconómico, edad y género. Para ello se diseñó un formato en el que los participantes evaluaban si cada ítem era comprensible, poco comprensible o nada comprensible. La muestra estuvo conformada por 380 personas, asumiendo valores de confianza de $95 \%$ y un error del 5\%. El grado de comprensibilidad de los ítems se determinó por medio de porcentajes obtenidos así: igual o superior al $85 \%$ : alta comprensibilidad; $80-85 \%$ mediana comprensibilidad; menor del 80\%: baja comprensibilidad. A partir de los resultados de la prueba se realizó el ajuste semántico requerido y una nueva prueba con un grupo de 20 personas con EC.

5) Pruebas de validez facial y de contenido con expertos. Con el fin de determinar si la versión ajustada semánticamente del instrumento realmente medía lo que se proponía se recurrió a la validez facial y de contenido con un panel de ocho expertos cuyos criterios de inclusión fueron tener posgrado en salud a nivel de especialización, maestría o doctorado y contar con 10 o más años de experiencia trabajando en el campo del cuidado a personas con enfermedad crónica o áreas afines. Se contó además con la asesoría de dos profesores de estadística con Maestría en este campo y una psicóloga experta en psicometría.

Los expertos revisaron los ítems en su coherencia, suficiencia, claridad, relevancia e importancia de acuerdo a lo propuesto por Lawshe [20] modificado por Tristan [21] Para determinar el grado de acuerdo entre jueces se utilizó el índice de concordancia Kappa de Fleiss [22], considerando la fuerza de concordancia como aceptable cuando los índices kappa se encontraran entre 0.41 y 0.60 y buena o muy buena si estos daban valores mayores a 0.61 . Por último para determinar el valor del índice de Contenido del instrumento (CVI) se consideró su medición global y por ítems.

\section{Resultados}

1. Desarrollo del instrumento. El instrumento Carga de la Enfermedad Crónica para el Paciente - GCPC-UN consta de 49 ítems distribuidos en tres dimensiones: el sufrimiento psicológico y espiritual, con 15 ítems; el disconfort y malestar físico, con 19 ítems; y la alteración socio cultural y familiar con 15 ítems. Cada situación que se asocia con la enfermedad es medida en términos de la frecuencia. (Tabla 1 que se anexa)

2. Las pruebas de comprensibilidad aplicadas a 380 personas del común reportaron comprensibilidad alta (superior al 85\%) para 35 ítems, moderada (80 a $84 \%)$ para 6 ítems y baja $(70,2 \%$ a $79,4 \%)$ para 6. Con estos hallazgos se procedió a una revisión de los ítems con moderada y baja comprensibilidad, a cargo de un filólogo. La versión ajustada semánticamente se aplicó a 20 personas del común obteniéndose una comprensibilidad alta para todos los ítems del instrumento.

3. Validez facial y de contenido

En el proceso de validez facial y de contenido se obtuvo una fuerza de concordancia entre moderada y sustancial (alta) por parte de los jueces, lo que permite afirmar que el instrumento es suficiente, claro, coherente y relevante en la medición de la carga de la enfermedad crónica para personas con enfermedad crónica.

Como observaciones adicionales, los expertos sugirieron incluir medición del ítem en criterios de intensidad y amenaza que representa para el usuario, teniendo en cuenta que la mayor parte de estos corresponden a síntomas que pueden estar presentes de forma permanente en los pacientes y la carga se relaciona con la preocupación que les representa y la intensidad o severidad referida. Así mismo, indicaron la necesidad de adicionar una pregunta final que permitiera a los usuarios describir un síntoma que no estuviese incluido en el instrumento, como auto-reporte. Se consideró que el 
instrumento puede ser auto diligenciado o aplicado por un clínico o un investigador.

La obtención del índice de contenido se basó en el modelo de Lawshe modificado [21], que representa una propuesta de comprobación útil para la valoración de la validez de contenido de las pruebas diseñadas, adaptadas o utilizadas en salud. En cada ítem se calculó la razón de validez de contenido modificada (CVR') y para todo el instrumento se obtuvo el índice de validez de contenido (CVI).

El CVI del instrumento en su medición global fue de 0,92, para los ítems se obtuvieron índices entre 0,62 y 1,00, hallazgo que indica que la validez de contenido en general es buena. (Tabla Anexo)

\section{Discusión}

Es sabido que comprender integralmente la experiencia física y emocional de las personas en condición de cronicidad cuando se busca asumir el cuidado es fundamental de acuerdo a lo referido por Montalvo [23], constituyéndose este como el primer paso en la valoración y planeación de la atención.

Aunque existen herramientas para valorar múltiples expresiones de la EC tales como el dolor [24, 25], La funcionalidad [26,27], el autoconcepto [28], la relación entre aspectos biológicos y psicológicos $(29,30)$, la condición espiritual (31), la capacidad de descansar [32,33], la situación económica [34,35], e incluso el nivel de apoyo social que el paciente recibe junto con su cuidador(36), hay también evidencia de que muchos de los síntomas y quejas del paciente con EC se quedan sin valorar [37] y que la valoración quizá por ello se ha enfatizado en la necesidad de tener en cuenta la perspectiva subjetiva del paciente [38], con base en la cual se abren importantes posibilidades para el cuidado de su salud [39].

Si bien se conocen algunos intentos de valorar de manera integral al paciente con EC como aquellos que siguen un esquema de patrones funcionales [40], los que lo caracterizan desde el punto de vista del cuidado [41\} y los que miran los diversos aspectos relacionado con la rehabilitación [42], la propuesta del presente estudio con el diseño y validación facial y de contenido del instrumento Carga de la Enfermedad Crónica para el Paciente - GCPC-UN genera un complemento necesario en este campo.

De igual forma complementa estudios con respecto a la carga del cuidado para el sistema [43-45], para la familia [46] y para el cuidador familiar [47], involucrando al sujeto directamente implicado en la experiencia de vivir con una enfermedad crónica.

El Instrumento Carga de la Enfermedad Crónica para el Paciente - GCPC-UN permite valorar integralmente el impacto que la enfermedad crónica genera desde el punto de vista del individuo afectado. Su desarrollo de comprensibilidad y validez, avala su exploración en el contexto colombiano e invita a continuar con nuevas pruebas psicométricas que garanticen mayor validez y confiabilidad.

Teniendo en cuenta estos hallazgos, se espera dar continuidad al proceso de validez y confiabilidad del instrumento por medio de:1) Validez de constructo utilizando técnicas de análisis factorial que permitan estimar el aporte de un ítem a una o varias dimensiones simultáneamente, 2) Confiabilidad: estableciendo la estabilidad y equivalencia de la escala con otras. 3) Especificidad para enfermedades crónicas como el cáncer 4) Sensibilidad, identificando la habilidad del instrumento para distinguir cambios en la percepción de carga con una intervención que busque el fortalecimiento de la misma.

\section{Conflicto de interés}

Los autores manifiestan no tener ningún conflicto de interés

\section{Referencias}

1. Marengoni A, Angleman S, Melis R, Mangialasche F, Karp A, Garmen A, Meinow B, Fratiglioni L. Aging with multimorbidity: a systematic review of the literature. Ageing Res Rev. 2011;10(4):430-9.
2. Eckerblad J, Theander K, Ekdahl A, Unosson M, Wirehn AB, Milberg A, et al. Symptom burden in community-dwelling older people with multimorbidity: a cross-sectional study. BMC geriatrics. 2015.15(1):1.

3. Embuldeniya G, Veinot P, Bell E, Bell M, Nyhof-Young J, Sale JE, et al. The experience and impact of chronic disease peer support interventions: A qualitative synthesis. Patient education and counseling. 2013; 92(1): 3-12.

4. Berwick DM, Nolan TW, Whittington J. The triple aim: Care, health, and cost. Health Aff. 2008;27(3):759-69.

5. Marks JS. Health-related quality of life reveals full impact of chronic diseases. Centers for Disease Control and Prevention. 2003;16(1):1-36.

6. Vinaccia S, Orozco LM. Aspectos psicosociales asociados con la calidad de vida de personas con enfermedades crónicas. Perspectivas en Psicología. 2005; 1(2): 125 - 137

7. Peláez E, Acosta L, Carrizo E. Factores asociados a la autopercepción de salud en adultos mayores. Revista Cubana de Salud Pública. 2015;41(4):638-648.

8. Lejarraga A. La construcción social de la enfermedad. Arch. argent. pediatr. [Internet] 2004 Ago [Consultado 2016 Feb 26]; 102(4): 271-276. Disponible en: http://www.scielo.org.ar/scielo. php?script=sci_arttext\&pid=S0325-00752004000400007\&lng=es.

9. Jackson D, McCrone P, Turner L. Costs of caring for adults with long-term neurological conditions. J Rehabil Med. 2013; 45(7): 653-61.

10. Arechabala, MC, Catoni MI, Barrios S, Palma E. Validación al español de la escala de auto percepción de carga de cuidado. Acta paul. enferm [Internet]. 2012 [Consultado 2016 Feb 26]; 25(1):140-145. Disponible en: http://dx.doi.org/10.1590/S010321002012000100024.

11. McPherson C, Wilson K, Murray MA. Feeling like a burden: Exploring the perspectives of patients at the end of life. Soc Sci Med. 2007;64(2):417-27.

12. Cousineau N, McDowell I, Hotz S, Hebert P. Measuring chronic patients' feelings of being a burden to their caregivers: Development and preliminary validation of a scale. Med Care. 2003;41(1):110

13. Srivastava K, Thakur D, Sharma S, Punekar YS. Systematic review of humanistic and economic burden of symptomatic chronic obstructive pulmonary disease. PharmacoEconomics. 2015;33(5): 467-488.

14. Dang-Tan T, Ismaila A, Zhang S, Zarotsky V, Bernauer M. Clinical, humanistic, and economic burden of chronic obstructive pulmonary disease (COPD) in Canada: a systematic review. BMC research notes. 2015; 8(1): 464.

15. Haworth SK, Dluhy NM. Holistic symptom management: modelling the interaction phase. Journal of advanced nursing. 2001; 36(2): 302-310.

16. Ganong LH. Integrative Reviews of Nursing. Rev Nurs Health. 1987; 10(1):1-11.

17. Sparbel KJH, Anderson MA. Integrated literature review of continuity of care: Part 1, conceptual ideas. Journal of Nursing Scholarship. 2000; 32 (1): 17-24.

18. Lenz E, Pugh LC. Theory of Unpleasant Symptoms,. En: Smith M, Liehr P (Editores), Middle Range Theory for Nursing. New York (USA);2008. p. 159 - 182.

19. Humphreys J, Kathyrin L, Carrieri V, Kathleen P, Julia F, Susan J, et al. Theory of symptom management . En Smith M, Liehr P (Editores) Middle Range Theory for Nursing. New York (USA); 2008. New York. P. 145-159

20. Lawshe C. H. (1975). A quantitative approach to content validity. Personnel Psychology. 28(4), 563-575. 
21. Tristán A. Modificación al modelo de Lawshe para el dictamen cuantitativo de la validez de contenido de un instrumento objetivo . Avances en Medición [Internet]. 2008 [ Consultado 2016 Feb 26) 6: 37-48. Disponible en : http://www.humanas.unal.edu.co/ psicometria/files/8413/8574/6036/Articulo4_Indice_de_validez_ de_contenido_37-48.pdf

22. Fleiss J. Measuring nominal scale agreement among many raters. Psycological bulletin. 1971; 76(5):378-382.

23. Montalvo A, Cabrera B, Quiñones S. Enfermedad crónica y sufrimiento: revisión de literatura. Aquichan. 2012 Agosto; (12)2: 134-143.

24. Díaz Puebla . Tipos de dolor y escala terapéutica de la O.M.S.: Dolor iatrogénico. Oncología (Barc.) [Internet]. 2005 Mar [Consultado 2015 Feb 20] ; 28(3): 33-37. Disponible en: http://scielo.isciii.es/scielo.php?script=sci_arttext\&pid=S037848352005000300006\&lng=e

25. Rodríguez MA, Hernández D, Gutiérrez MJ, Francisca JA, Calls Ginesta Jordi. Evaluación del dolor crónico en una población de pacientes hemodializados. Rev Soc Esp Enferm Nefrol [Internet]. 2007 Jun [Consultado 2015 Feb 20] ; 10(2): 65-71. Disponible en: http://scielo.isciii.es/scielo.php?script=sci_arttext\&pid=S1139$13752007000200005 \& \operatorname{lng}=$ es.

26. Cid J, Damián J. Valoración de la discapacidad física: el indice de Barthel. Rev. Esp. Salud Publica [Internet]. 1997 Mar [Consultado 2015 Feb 20] ; 71(2): 127-137. Disponible en: http://scielo.isciii.es/scielo.php?script=sci_arttext\&pid=S113557271997000200004\&lng=es.

27. Webster K, Cella D, Yost K . The F unctional A ssessment of C hronic I llness T herapy (FACIT) Measurement System: properties, applications, and interpretation. Health and Quality of Life Outcomes [Internet]. 2003 [Consultado 2015 Feb 20]; 1(1):79. Disponible en: http://hqlo.biomedcentral.com/ articles/10.1186/1477-7525-1-79\#Abs1. DOI: 10.1186/14777525-1-79.

28. Cercós A, Beniel D, Pérez M, Alcón JJ, Prado VJ. El autoconcepto y la adaptación a la enfermedad en pacientes diabéticos pediátricos. Clínica y Salud. 2004. 25( 1): 57-65

29. Soucase B, Monsalve V, Soriano J. F. Afrontamiento del dolor crónico: el papel de las variables de valoración y estrategias de afrontamiento en la predicción de la ansiedad y la depresión en una muestra de pacientes con dolor crónico. Rev. Soc. Esp. Dolor [ Internet]. 2005 Feb [Consultado 2015 Feb 21] ; 12(1): 8-16. Disponible en: http://scielo.isciii.es/scielo.php?script=sci_ arttext\&pid=S1134-80462005000100003\&lng=es.

30. Rodríguez M, Merino $\mathrm{M}$, Castro $\mathrm{M}$. Valoración psicométrica de los componentes físicos (CSF) y mentales (CSM) del SF36 en pacientes insuficientes renales crónicos en tratamiento con hemodiálisis. Cienc. enferm. [Internet]. 2009 Abr [Consultado 2015 Feb 11]; 15( 1 ): 75-88. Disponible en: http://www.scielo.cl/scielo.php?script=sci_arttext\&pid=S071795532009000100009\&lng=es. http://dx.doi.org/10.4067/S071795532009000100009.

31. Ottaviani AC, Souza EN, Camargo N, Zazzetta MS, lost- Pavarini SC, De Souza F. Esperanza y espiritualidad de pacientes renales crónicos en hemodiálisis: un estudio de correlación. Rev. LatinoAm. Enfermagem. 2014;22(2):248-54

32. Varela LF, Tello T, Ortiz PJ, Chávez H. Valoración de la higiene del sueño mediante una escala modificada en adultos mayores. Acta Médica Peruana [Internet]. 2010 [ Consultado 2015 Feb 10];27(4): 233-237. Disponible en http://www.scielo.org.pe/scielo. php?script=sci_arttext $\&$ pid $=$ S1728-59172010000400005\&lng=es \&nrm=iso.
33. López F, Rodríguez OF , Agüeroa LF , Mareque MA, Lázaro GA, Montillaa JB. Valoración Clínica y Calidad de Vida relacionada con la Salud del Paciente con Insomnio. Rev clín med fam. 2011; 4 (2): 92-99.

34. Essue B, Kelly P, Roberts M, Leeder S, Jan S. We can't afford my chronic illness! The out-of-pocket burden associated with managing chronic obstructive pulmonary disease in western Sydney, Australia. J Health Serv Res Policy. 2011; 16(4): 226-231.

35. Lin JMS, Resch S, Brimmer DJ, Johnson A, Kennedy S, Burstein $\mathrm{N}$, et al. The economic impact of chronic fatigue syndrome in Georgia: direct and indirect costs. Cost Effectiveness and Resource Allocation. 2011; 9(1): 1 .

36. Suarez MA. Identificación y utilidad de las herramientas para evaluar el apoyo social al paciente y al cuidador informal. Rev. Méd. La Paz [Internet]. 2011 [Consultado 2015 Feb 12] ; 17( 1 ): 60-67. Disponible en: http://www.scielo.org.bo/scielo. php?script=sci_arttext\&pid=S1726-89582011000100010\&lng=es.

37. Palma A, del Río I, Bonati P, Tupper L, Villarroel L, Olivares P, et al . Frecuencia y pesquisa de síntomas en pacientes crónicos en fases avanzadas en un hospital clínico: ¿Existe concordancia entre pacientes y médicos?. Rev. méd. Chile [Internet]. 2008 Mayo [Consultado 2015 Ene 31] ; 136( 5 ): 561-569. Disponible en: http://www.scielo.cl/scielo.php?script=sci_arttext\&pid=S003498872008000500003\&lng=es. http://dx.doi.org/10.4067/S003498872008000500003

38. García V, Regina C, Rodríguez LG. Calidad de vida en enfermos crónicos. Rev haban cienc méd [Internet]. 2007. Nov [Consultado 2015 Feb 26]; 6(4). Disponible en: http:// scielo.sld.cu/scielo.php?script=sci_arttext\&pid=S1729519X2007000400002\&lng=es.

39. Cortés AR, Villarreal RE, Galicia RL, Martínez GL, Vargas ER. Evaluación geriátrica integral del adulto mayor. Rev. méd. Chile [Internet]. 2011 Jun [Consultado 2015 Ene 18] ; 139( 6 ): 725731. Disponible en: http://www.scielo.cl/scielo.php?script=sci_ arttext\&pid=S0034-98872011000600005\&lng=es. http://dx.doi. org/10.4067/S0034-98872011000600005.

40. Meizoso A, Sáez L, Valiño C, Gallego S, Seoane M. Guía de valoración del paciente crónico en hemodiálisis por indicadores Enfermería Nefrológica. Revista de la sociedad española de enfermería nefrologica. 2009. 12(4): 283-287.

41. Barrera L, Vargas E, Cendales PA. Encuesta de caracterización para el cuidado de una persona con enfermedad crónica. Investig. Enferm. Imagen Desarr. 2015 enero-junio; 17 (1): 27-43.

42. Casas E, Escandell MJ, Ribas M, Ochoa S. Instrumentos de evaluación en rehabilitación psicosocial. Revista de la Asociación Española de Neuropsiquiatría. 2010; 30(1): 25-47.

43. Abegunde DO, Mathers CD, Adam T, Ortegon M, Strong K. The burden and costs of chronic diseases in low-income and middleincome countries. Lancet. 2007; 370(9603):1929-1938.

44. World Bank. Brazil addressing the Challenge of Noncommunicable Diseases in Brazil. Brazil: World Bank; 2005 [ Consultado 2015 Feb 10] . 10 p.

45. Glassman A., Gaziano TA, Buendia CPB, de Aguiar FCG. Confronting the chronic disease burden in Latin America and the Caribbean.Health Affairs. 2010. 29(12); 2142-2148.

46. McRae I, Yen L, Jeon YH, Herath PM, Essue B. Multimorbidity is associated with higher out-of-pocket spending: a study of older Australians with multiple chronic conditions. Aust J Prim Health. 2013; 19(2): 144-9. 
47. Breinbauer H, Vásquez H, Mayanz S, Guerra C, Millán T. Validación en Chile de la Escala de Sobrecarga del Cuidador de Zarit en sus versiones original y abreviada. Rev. méd. Chile [Internet]. 2009 Mayo [citado 2014 Dic 11] ; 137( 5 ): 657-665. Disponible en: http://www.scielo.cl/ scielo.php?script=sci_arttext\&pid=S0034-98872009000500009\&lng=es. http://dx.doi.org/10.4067/S0034-98872009000500009.

Anexo

Tabla . Resultados validez facial y de contenido instrumento "carga de la enfermedad crónica para el paciente - GCPC-UN

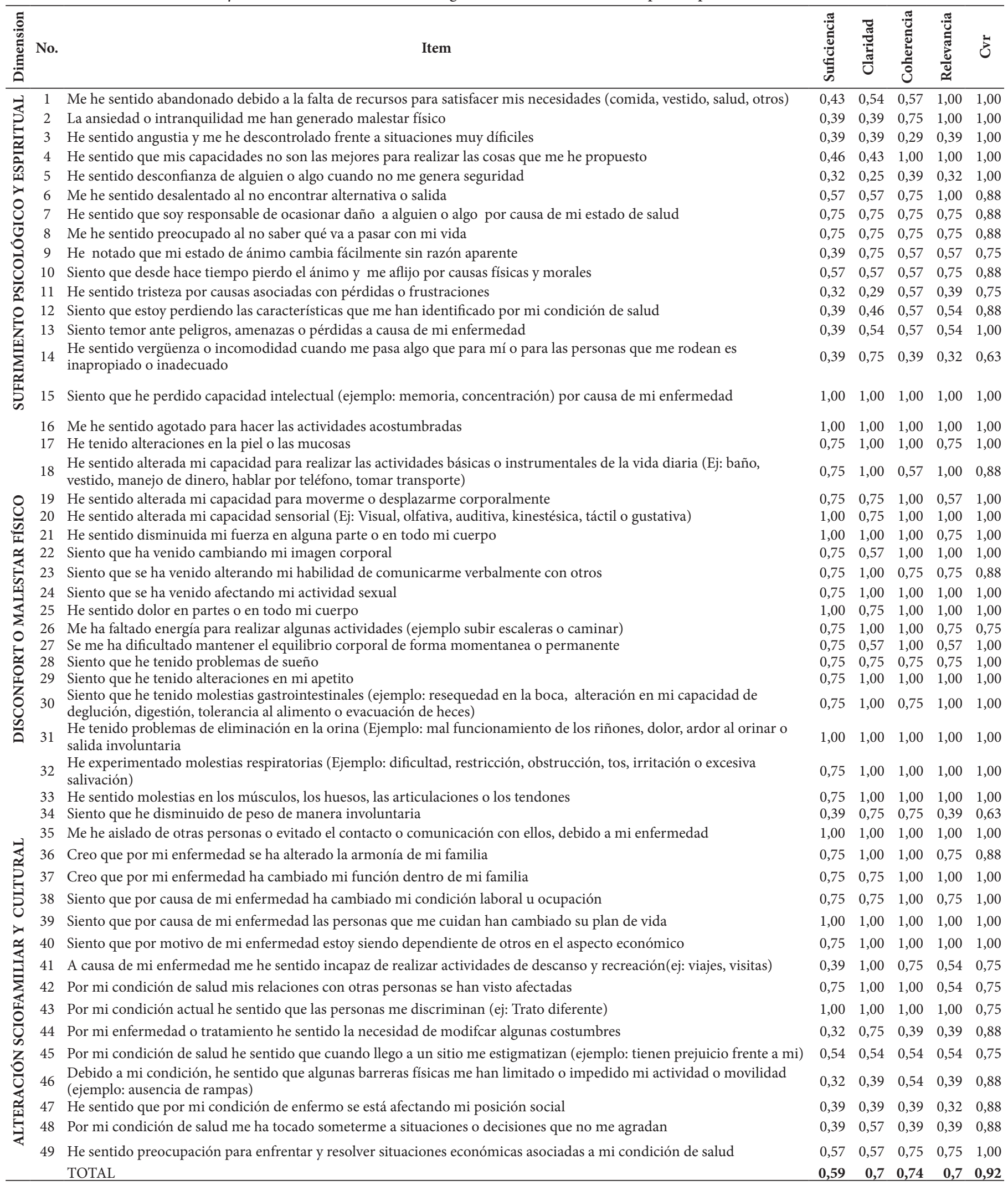

